\title{
THE EFFECT OF AWARENESS ON PERFORMANCE GAINS IN GONCEPT CONDITIONING
}

\author{
HAJIME YOSHIDA ${ }^{1,2}$ \\ Department of Educational Psychology, Kyushu University
}

\begin{abstract}
The study investigated the temporal relationship between performance gains and awareness in concept conditioning. The Ss were required to select 1 of the 4 words of 4 different concepts. Awareness of the concept and the contingency between responses and reinforcement was evaluated by means of a postconditioning interview. Successful conditioning was found in Ss who had acquired the concept. The performance increased after Ss acquired the concept, though performance in these Ss did not increase prior to reports of the concepts. Performance gains were not found in Ss who were merely aware of the contingency, nor in Ss who were not aware of the contingency. The results suggest that performance gains in concept conditioning are not mediated by awareness of the contingency, but mediated by awareness of the concept.
\end{abstract}

In early studies of verbal conditioning, Greenspoon (1955) and Taffel (1955) reported the experiments which appeared to demonstrate the autonomic unconscious effects of reinforcement on verbal behavior. Such effects have been accepted as evidence of learning without awareness (Greenspoon, 1962; Krasner, 1958). However, in experiments in which awareness was evaluated more thoroughly, the findings have suggested that performance gains in verbal conditioning are mediated by cognitive processes (Levin, 1961; Spielberger, 1962).

In order to know which of the theories worked better, DeNike (1964) examined the temporal relationship between performance and awareness by having subjects report their thoughts about the contingency in written notes at the end of

1 The author wishes to thank Professor Tatuo Endo and Associate Professor Mituya Yamauchi of Kyushu University for their guidance and criticism during the conduct of this study.

2 Requests for reprints should be sent to Hajime Yoshida, Department of Educational Psychology, Miyazaki University, 130 Nishimaruyama, Miyazaki 880 , Japan.

3 Now at Department of Educational Psychology, Miyazaki University. each block of 25 conditioning trials. $\mathrm{He}$ found that only subjects who were aware of the correct contingency showed performance gains, and the gains first occurred on the trial block at the end of which aware subjects first reported awareness. The similar result was reported by Spielberger, Bernstein, and Ratliff (1966). From these results it was concluded that awareness mediates performance gains in verbal conditioning (Spielberger \& DeNike, 1966). More recently, Kennedy (1971) assessed awareness every 10 trials and examined this relationship in both Greenspoon and Taffel type tasks. Kennedy found that aware subjects demonstrated performance gains prior to reports of awareness in both tasks and interpreted the results as supporting a behavioral explanation of verbal conditioning. However, the procedure to assess awareness during conditioning was criticized as prompting the acqusition of awareness (Kanfer, 1968). Attempting to reply to this criticism, Yoshida (1973a) devised a new method to identify the trial block on which awareness was acquired. In this method the trial block on which subjccts acquired awareness was evaluated by means of a postconditioning interview. He found that performance did not increase 
before the trials on which awareness was acquired.

Yoshida (1973b) pointed out that what is learned in the Taffel type task differs from that of the Greenspoon type task. What is learned in the Taffel type task is the relationship between responses and reinforcement, while what is learned in the Greenspoon type task is response patterns of two types. The first is the type that subjects emit each response without being aware of the concept. The second is the type that subjects emit each response after acquiring the concept itself and realizing it be relevant. In the former subjects emit associative response, while in the latter subjects emit conceptual response. Accordingly, Yoshida denoted this latter type of conditioning as "concept conditioning." The concept used here refers to common attribute of events. In his study, Yoshida conducted the experiment in which concept conditioning is possible even in the Taffel type task and tried to differentiate between subjects making associative response and conceptual response. The subjects were divided into three groups; subjects who were unaware of the contingency (unaware subjects), subjects who were only aware of the correct contingency (associative subjects), and subjects who acquired the concept (conceptual subjects). Examination of performance curves of these groups revealed that there were significant performance increments in conceptual subjects but not in associative subjects nor in unaware subjects. From Yoshida's study, it became clear that aware subjects should be divided into conceptual and associative subjects.

The purpose of the present study was to examine the temporal relationship between performance gains and awareness in both conceptual subjects and associative subjects. Awareness of each subject and the trial block on which awareness was acquired was assessed by means of a detailed postconditioning interview.

\section{Method}

Subjects

The subjects were 41 high school students in Fukuoka city. None of the subjects had previously participated in a psychological experiment. Experimenters were five male graduate students.

\section{Stimulus materials}

The materials in the present experiment were same as were used in R-3 of four conditions in the previous study (Yoshida, 1973b). The same 12 words were used as stimulus words. For the details of these materials, refer to Yoshida's previous work (1973b). The words in each category were as follows.

noun：本だな (bookcase), 机 (desk), ひきだ ᄂ (drawers)

verb : 走る (run), 歩く (walk), 飛ぶ (jump) adjective：大きい (big), 強い (strong), 頼 む しい(robust)

word of foreign origin: ケーキ(cake), レモ ン (lemon), タリーム (cream)

The stimulus materials consisted of $80,18 \times 13$ $\mathrm{cm}$ plain white strips of paper. On each card four words werc printed; one word from each category. The position of appearance of each category and the appearance order of each word from a category were randomized with the restriction that no same word appeared successively on all cards. All stimulus words used in 1st-30th trials were written in black color. The 31 st- 80 th trial were divided into five blocks of 10 trials each, and the stimulus words in each block were written in one of five colors : red, blue, green, brown, and yellow. The order of color in five blocks was randomized among subjects.

\section{Instruction}

The experimenter read the following instruction, showing a practice card to subject: "We are doing a study on how people use words. Your job today will be very simple. I want you to select a word from four words written on this card. This is a practice. Which word would do you select?" Then, 
subject was instructed to continue in the same manner when he was given the next card.

\section{Conditioning procedure}

The subject's task was to select one of four words written on a card. After completing 20 operant trials, an operant level to each category was immediately counted in order to determine the critical category, which was of the highest operant level. When the first and second operant level had same frequencies, experimenter chose randomly one of two categories. Of 41 subjects, two subjects were excluded from the latter part of the experiment, because they responded to only a category during the operant period. Each time subject used a word from the category which was of the highest operant level during 60 conditioning trials, he was reinforced with a mild affirmatory word ("good"). When subject selected a word from the other three categories, he was not reinforced.

\section{Method for assessing awareness}

After completion of the conditioning task, each subject was directed to another room and interviewed to classify him into one of three groups: subjects who acquired the concept, subjects who did not acquire the concept but were aware of the contingency between responses and reinforcement, and subjects who unaware of the correct contingency. The interview questions used were as follows.

1. Were you aware that I said something when you selected the word? yes, no

2. What did I say? ( )

3. Did you know when I said so? (If subject gave the correct concept, question 6 followed question 3 . If subject correctly reported more than one word, question $4,5,6$ followed question 3.)

4. Were you aware of any common feature about their words? yes, no

5. What common feature was there among their words? ( )

6. Were you aware of the feature because I asked you to report it? yes, no

7. What was the color when you were aware of the feature? ( )
The criteria to classify subjects into three groups from a postconditioning interview were as follows. A criterion of conceptual subjects was the report of common attribute among responses. A criterion of associative subjects was the report of correct contingency. The subjects who reported the common attribute but answered "yes" to question 6 were classified as associative subjects, because they were not aware of the concept during the conditioning trials. The subjects who were not aware of the correct contingency and the reinforcement itself were classified as unaware subjects.

\section{Results}

\section{Classification of subjects}

Three raters who had no knowledge of subject's performance data assessed each subject's responses at a postconditioning interview and classified him into one of three groups. Three subjects for whom there was the disagreement of assessment among three raters were classified into one of three groups after discussion among raters. By such a procedure, 14 subjects were classified as conceptual subjects, 10 subjects as associative subjects, and 15 subjects as unaware subjects. One of conceptual subjects was eliminated from the final data because he could not point out the specific color to question 7. Of the concepts identified by subjects, nine subjects identified the concept like noun, verb, adjective, or word of foreign origin. There were three subjects who described semantic concepts as "food" or "something to study." One subject who was reinforced for verb answered "foot" as common attribute. $\mathrm{He}$ was judged to have acquired the concept because the verbs like " run," " walk," or " jump," were related to foot.

\section{Performance of conceptual, associative, and unaware subjects}

For subsequent analysis the trials were divided into four blocks of 20 trials each. Fig. 1 shows the mean frequencies of each 
group over the four blocks of trials. An analysis of variance for the operant trial block indicated that there was no significant difference among groups, $F(2,35)=$ 1.094. A repeated-measures analysis of variance for the performance data revealed that there was significant difference among groups, $F(2,35)=14.925, \quad p<.001$; that there was a significant trial blocks effect, $F(3,105)=4.354, p<.01 ;$ and that the Groups $\times$ Trial Blcoks interaction was also significant, $F(6,105)=7.568, p<.001$. Because the Groups $\times$ Trial Blocks interaction was significant, $t$ tests were computed between the means of the operant and 3 blocks for the three groups. These comparsions were significant in conceptual subjects, $t(12)=5.342, p<.001$, but not in associative subjects, $t(9)=.703$, nor in unaware subjects, $t(14)=.864$.

Performance of conceptual and associative subjects prior to and post reports of awareness

The words were written by different colors among six blocks of 10 trials each in acqusition trials. Twenty operant trials were divided into two blocks of 10 trials each and over all trials were divided into eight blocks of 10 trials each. In order

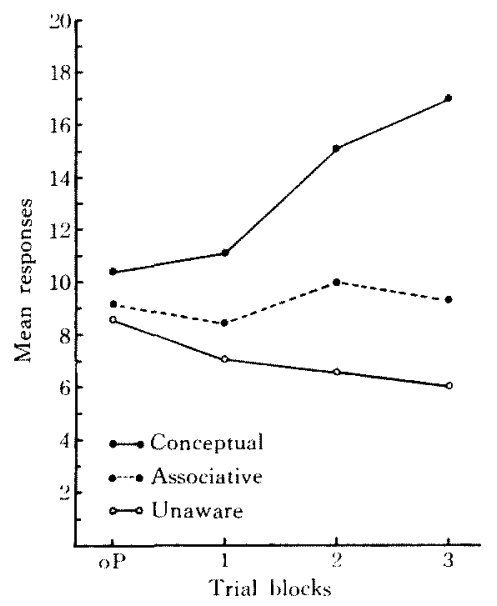

Fig. 1. Group performance curves of conceptual, associative, and unaware groups. to examine whether performance increased prior to the trial block on which the concept was acquired, the trial block on which subjects acquired the concept was assessed from their responses to a postconditioning interview. The trial block of color which subjects answered to question 7 was determined as the trial block on which the concept was acquired.

Since subjects reported the concept at different times, the performance of conceptual subjects was analyzed as a function of the trial block on which each subject reported the concept. To accomplish this, performance data were arrayed so that the trial block on which each subject reported the concept were aligned and designated as the " 0 " trial block. Then, preverbalization trial blocks were labeled as "preaware" and postverbalization trial blocks as "postaware." The number of subjects in each trial block were as follows. Subjects in " -5, ," " -4 ," " -3, , " -2, , " -1, , “ 0 ," " 1, , " 2 ,", " 3 ," " 4 ," " 5 ," trial block were 2, 2, 10 , $13,13,13,13,13,11,11,3$, respectively. The mean frequencies over each block are presented as raw data curve in Fig. 2. The data for the preaware trial blocks were then Vincentized to three blocks and for the postaware, including " 0 " block, to five blocks so that each subject's performance contributed to each data point. This Vincentized data curve is shown in Fig. 2. Similar procedure was followed on the performance curve of associative subjects. The numbers of subjects in " -5 ," " -4, , " -3, ," " -2, , " " -1, , " “ 0, , " " 1 ,", " 2 ," " 3 ," " 4 ," block were $1,5,8,10$, $10,10,10,10,9,6$, respectively. The mean frequencies over each block are raw data curve in Fig. 3. The data for the preaware trial blocks were Vincentized to four blocks and for the postaware, including " 0 " block, to five blocks. This Vincentized data curve is shown in Fig. 3.

As can be seen in Fig. 2 and Fig. 3, the raw data curve, which is based upon a varying number of data entries for each 
trial block, is similar to the Vincentized data curve. In order to evaluate the temporal relationship between performance gains and awareness, the Vincentized curves in both preaware and postaware blocks in Fig. 2 were subjected to a repeated-measures analysis of variance. This analysis yielded that there were no significant performance increases in preaware blocks, $F(2,24)=1.074$, but significant performance increases in postware blocks, $F(4,48)=3.616, \quad p<.025$. Similarly, a repeated-measures analysis of variance for the Vincentized data in Fig. 3 indicated that there were no significant performance increases in preaware blocks,

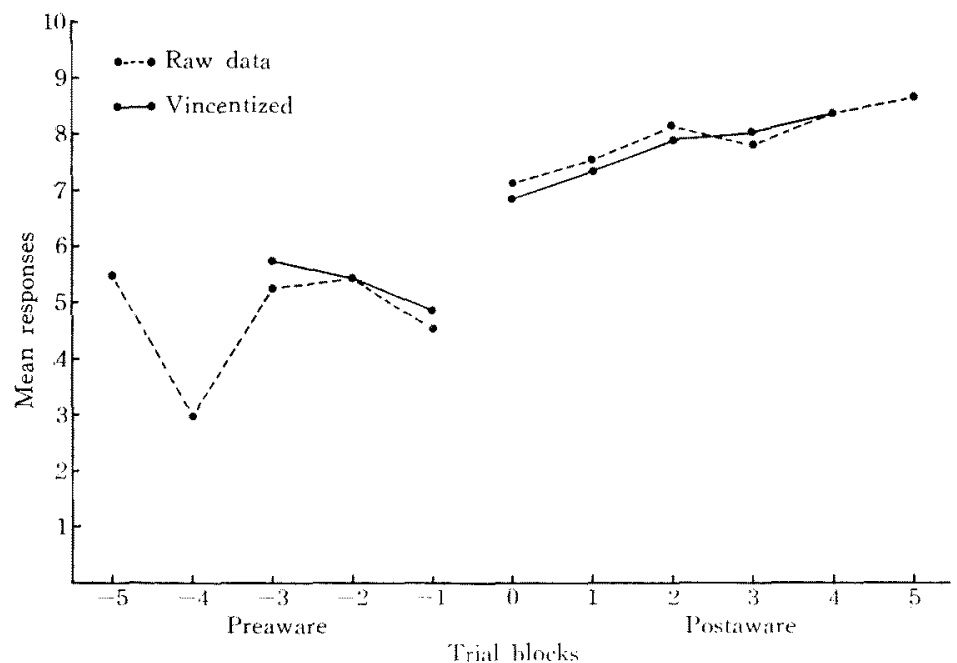

FIG. 2. Vincentized and raw data curves for conceptual group who reported the concept on trial block " $0 . "$

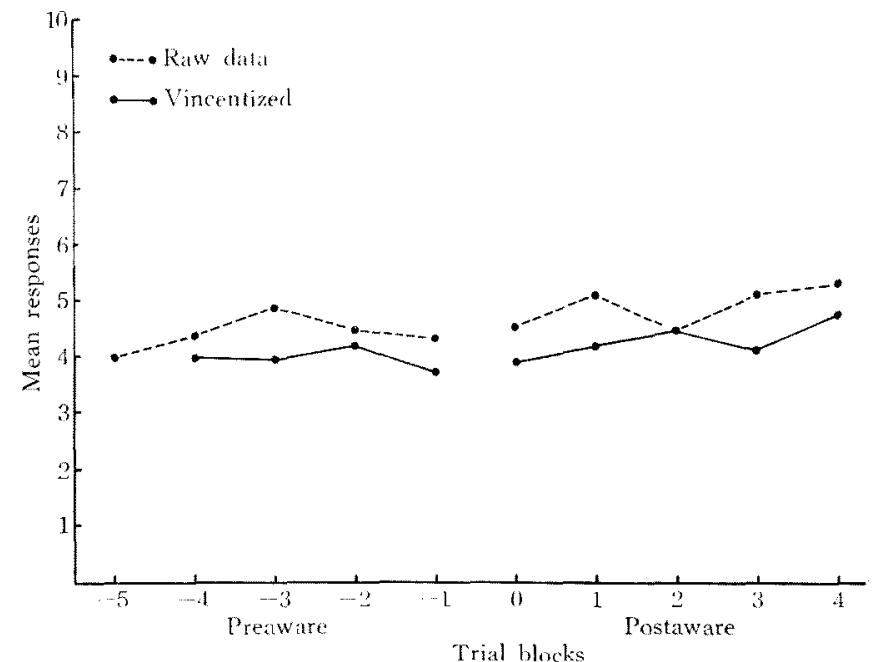

Fig. 3. Vincentized and raw data curves for associative group who reported the contingency on trial block " $0 . "$ 


\section{TAble 1}

The difference between " -1 " and " 0 " trial block in Vincentized and raw data for conceptual group

\begin{tabular}{lccc}
\hline & Trial block & \\
& -1 & 0 & \\
& -1 & & \\
& 4.7 & 7.1 & 2.4 \\
Raw data & 4.9 & 6.9 & 2.0
\end{tabular}

TABLE 2

The difference between " -1 " and " 0 " trial block in Vincentized and raw data for associative group

\begin{tabular}{lccc}
\hline & \multicolumn{2}{c}{ Trial block } & \\
& -1 & 0 & \\
& -1 & & \\
& 4.2 & 4.5 & 0.3 \\
Raw data & 3.5 & 3.9 & 0.4
\end{tabular}

$F(3,27)=.927$, nor in postaware blocks, $F(4,36)=1.546$.

Performance gains due to acquiring the concept or contingency

Learning theories which posit the direct and automatic strengthening of response by reinforcement would predict gradual increments in performance over trial (Postman \& Sassenrath, 1961). However, on the assumptions that conceptual and associative subjects developed their awareness during " 0 " trial block and began promptly to select their response on the basis of these awareness, it would be expected that performance gains would begin on the " 0 " trial block. In order to test this expectation, the responses between " -1 " and " 0 " block were compared. The mean frequencies for each block of conceptual subjects are presented in Table 1. It was found that performance on the " 0 " trial block was significantly above performance on the " -1 " trial block for both the raw data, $t(12)=2.184, p<.05$, and the Vincentized data, $t(12)=2.415$, $p<.005$. The mean frequencies for each block of associative subjects are presented in Table 2. It was also found that performance on the " 0 " block was not above performance on the " -1 " block for the raw data, $t(9)=1.006$, and the Vincentized data, $t(9)=-.948$.

\section{Discussion}

In this investigation, the subjects who were only aware of the correct contingency did not show performance gains. Associative subjects correspond to aware subjects in the previous verbal conditioning studies. Although subjects who were aware of the contingency in the previous studies demonstrated performance increments, the present findings were not in accordance with the results of previous studies. Instead, subjects who acquired the concept demonstrated successful conditioning. These results were consistent with those of Yoshida's previous concept conditioning study (Yoshida, 1973b).

In the most of the previous verbal conditioning studies, it was implicitly assumed that cognitive process in the Greenspoon type task was the same as the one in the Taffel type task. However, the present results argue for the need for differentiation between concept conditioning and association conditioning in verbal conditioning. Therefore, the previous studies should be reassessed from point of view of the task used. In the experiment using the Taffel type task, Kennedy (1971) found that performance increased monotonically as a function of trials independent of the time in which subjects reported awareness during conditioning. Attempting to reply to criticism on the traditional method to assess awareness during conditioning, however, Yoshida (1973a) found that aware subjects did not show performance gains prior to aware block in the Taffel type task and interpreted his results as supporting cognitive position of verbal conditioning.

DeNike (1964) and Spielberger et al. 
(1966), who used the Greenspoon type task, demonstrated that a cognitive learning process mediates performance gains in verbal conditioning. However, Kennedy (1971) reported the result supporting a behavioristic position in the Greenspoon type task. But in Kennedy's experiment using this type task, the following results can be interpreted from cognitive position. Firstly, the performance of unaware subjects was unchanging from the operant base rate, while aware subjects showed clear performance gains. The response tendencies of these subjects are consistent with the results supporting cognitive position. Secondly, since Kennedy noted that 4 subjects of the 20 aware subjects received no reinforcement prior to the aware block, he excluded them from aware subjects. The performance of preaware blocks in the remaining 16 subjects was found to be significant. However, those who were aware of the contingency immediately after receiving reinforcement are subjects who have shown a cognitive process. Then, it can be argued that since the performance gains of preaware blocks in the 20 subjects were found to be insignificant, these data should be taken into account. Thirdly, the performance jumped discontinuously from preaware blocks to trial block on which subjects acquired awareness in the Greenspoon type task. Such results are consistent with DeNike's findings. Thus, from these facts it can be argued that Kennedy's study using the Greenspoon type task supports the cognitive explanation of verbal conditioning.

From reexaminations for previous studies, it can be expected that conceptual subjects do not show significant performance gains prior to the time at which subjects acquired the concept. As can be seen in Fig. 2, performance gains in preaware blocks were not significant. It is clear that only the result of nonsignificant effect in preaware blocks can not guarantee the discontinuous gains of performance as it is expected from the cogni- tive view. Thus, the responses between " -1 " and " 0 " block on conceptual subjects were compared. Performance at " 0 " block was significantly above the performance at " -1 " block.

The process through which conceptual subject showed the discontinuous gains of performance might be considered to be as follows: (a) Subjects become aware that reinforcements were given to one or two of all responses. (b) Then, subjects elicit internally the concept from the responses. (c) By using this concept as mediator, the other responses are conditioned at a stretch also. This process might be like that of clustering suggested by Bousfield (1953).

What is expected from associative subjects? As associative subjects are aware of the correct contingency, they are considered to be in same state as aware subjects in the Taffel type task. In this type task subjects who were aware of the contingency demonstrated performance increments as most of the previous studies confirmed (Taffel, 1955; Yoshida, 1973a). Therefore, it is expected that associative subjects who were aware of the correct contingency show performance gains, and the gains first occur on the " 0 " trial block. However, as can be seen in Fig. 1 and Fig. 3, there were no performance gains over trial blocks and no significant difference between " -1 " and " 0 " block for the raw and the Vincentized data. Then, why don't associative subjects show the performance gains by being aware of the contingency? Such a discrepancy between the results of the previous experiments and those of the present may be due to different experimental tasks used. Unfortunately, the sufficient data to this question is not available to us.

In the most of the previous studies the trial block on which awareness was acquired was evaluated by having subjects write notes during the conditioning trials. It was a marked advance that this method made directly the state of response prior 
to and post reports of awareness clear. However, the method to assess repeatedly awareness during the conditioning trials was criticized as providing opportunity for acquiring awareness (Kanfer, 1968) and it have driven this problem into unsolvable dilemma. The new method used in the present experiment to assess after the conditioning trials the block on which awareness is acquired makes a thorough reply to this criticism. Advantage of this new method is also observed in the following results. In some previous studies, it was observed that there were no performance gains in postaware trial blocks. On this problem, hypothesizing that this low rate was due to lack of incentive, Spielberger et al. (1966) instructed all subjects after the seventh block of trials that there was a rule for reinforcement, and subjects were to try to receive many reinforcements as possible. For subjects who had been aware before these added instructions, performance jumped from approximately $30 \%$ emission to about $55 \%$. It should be emphasized, however, that in the present experiment the jump of performance was ascertained by using the method that the experiment was conducted without interrupting the conditioning task and telling instruction which appears to enhance the incentive value.

\section{REFERENCES}

Bousfield, W.A. 1953 The occurrence of clustering in the recall of randomly arranged associates. Journal of General Psychology, 49, 229-240.

DeNIKE, L. D. 1964 The temporal relationship between awareness and reinforcement in verbal conditioning. Fournal of Experimental Psychology, 68, 521-529.

Greenspoon, J. 1955 The reinforcing effect of two spoken sounds on the frequency of two responses. American fournal of Psychology, 68, $409-416$.
Greenspoon, J. 1962 Verbal conditioning and clinical psychology. In A.J. Bachrach (Ed.), Experimental foundations of clinical psychology. N.Y.: Basic Books, Pp. 510-553.

KANPER, F.H. 1968 Verbal conditioning: $\Lambda$ review of its current status. In T. R. Dixon \& D. L. Horton (Eds.), Verbal behavior and general behavior theory. Englewood Clifs, N.J.: Prentice Hall.

Kennedy, T. D. 1971 Reinforcement frequency, task characteristics, and interval of awareness assessment as factors in verbal conditioning without awareness. Fournal of Experimental Psychology, 85, 103-112.

KRASNER, L. 1958 Studies of the conditioning of verbal behavior. Psychological Bulliten, 55, 148-170.

Levin, S. M. 1961 The effects of awareness on verbal conditioning. Journal of Experimental $P_{\text {sychology }}, 61,67-75$.

Postman, L., \& Sassenrath, J.M. 1961 The automatic action of verbal rewards and punishments. Journal of General Psychology, 65, 109136.

Spielberger, C. D. 1962 The role of awareness in verbal conditioning. In C.W. Eriksen (Ed.), Behavior and awareness. Durham: Duke University Press, Pp. 73-101.

Spielaerger, C. D., \& DeNike, L. D. 1966 Descriptive behaviorism versus cognitive theory in verbal operant conditioning. Psychological Review, 73, 306-326.

Spielaerger, C. D., Bernstein, I. H., \& RatlifF, R. G. 1966 Information and incentive value of the reinforcing stimulus in verbal conditioning. Journal of Experimental Psychology, 71, 2631.

TAFFEL, C. 1955 Anxiety and the conditioning of verbal behavior. Journal of Abnormal and Social Psychology, 51, 496-501.

YoshidA, H. 1973a The effect of awareness on the performance gains in verbal conditioning. The fapanese fournal of Psychology, 44, 135-140. (In Japanese with English Summary)

YoshidA, H. 1973b The distinction of two types in verbal conditioning: "concept" conditioning and "association" conditioning. The Japanese Journal of Psychology, 44, 241-249. (In Japanese with English Summary)

(Received Feb. 12, 1975) 